\title{
Existence of Periodic Solutions for the Quasi-Static Thermoelastic Thermistor Problem
}

\author{
Maurizio Badii
}

\begin{abstract}
We deal with a new model for the thermistor problem formulated as a coupled system of PDE's involving nonlinear energy heat equation, stationary charge conservation equation of electrical current and thermoelastic equations of displacement. We establish the existence of weak periodic solutions rewriting our system as an abstract problem in order to utilize the maximal monotone mappings theory and a fixed point argument for a suitable operator equation.
\end{abstract}

Mathematics Subject Classification (2000). 35B10, 35D05, 35K55, 35M10.

Keywords. Periodic solutions; thermistor problem; quasi-static thermoelastic equations; weak solutions.

\section{Introduction}

The aim of this paper is to study the existence of weak periodic solutions for a new model for the thermistor problem. The model consists of a nonlinear coupled system of partial differential equations which includes the evolution equation for the temperature in a conductor body with the Joule heating $\sigma(\theta)|\nabla \varphi|^{2}$ as a source, the stationary charge conservation equation of electrical current with temperature dependent electric conductivity $\sigma(\theta)$ and a system of quasi-static linear thermoelasticity equations of displacement subject to external forces and heat sources.

In what follows, the reference configuration of the thermistor $\Omega$ is an open, bounded and regular set of $R^{n}(n \geq 2)$ with boundary $\partial \Omega$ and the unit outward normal $\nu=\left(\nu_{1}, \ldots, \nu_{n}\right)$ is defined at points of $\partial \Omega$. We assume that the smooth boundary $\partial \Omega$ is divided into two relatively open parts $\Gamma_{D}$ and $\Gamma_{N}$ such that $\Gamma_{D}$ $\cap \Gamma_{N}=\emptyset$ and $\partial \Omega=\bar{\Gamma}_{D} \cup \bar{\Gamma}_{N}$. We denote by $P:=R / \omega Z$ the period interval $[0, \omega]$, so the functions defined in $Q$ are automatically time $\omega$-periodic. 
We may write the system as

$$
\left\{\begin{array}{c}
\theta_{t}-\sum_{i, j=1}^{n} \frac{\partial}{\partial x_{j}}\left(k_{i j} \frac{\partial \theta}{\partial x_{i}}\right)=\sigma(\theta)|\nabla \varphi|^{2}-\sum_{i, j=1}^{n} m_{i j} \frac{\partial^{2} u_{i}}{\partial t \partial x_{j}} \text { in } Q:=\Omega \times P, \\
\operatorname{div}(\sigma(\theta) \nabla \varphi)=0 \text { in } Q, \\
-\sum_{i, j, k, l=1}^{n} \frac{\partial}{\partial x_{j}}\left(a_{i j k l} \frac{\partial u_{k}}{\partial x_{l}}\right)+\sum_{i, j=1}^{n} \frac{\partial}{\partial x_{j}}\left(m_{i j} \theta\right)=f_{i} \text { in } Q,
\end{array}\right\}
$$

The $k_{i j}$ and $m_{i j}$ are the components of the heat conduction tensor and the thermal expansion tensor respectively, both assumed to be symmetric. The $a_{i j k l}$ are the components of the elasticity tensor, the symmetries of which are indicated below. Finally, $\mathbf{f}=\left(f_{1}, \ldots, f_{n}\right)$ is the density of the applied body forces. The boundary and periodic conditions for the problem are

$$
\begin{aligned}
-\sum_{i, j=1}^{n} k_{i j} \frac{\partial \theta}{\partial x_{i}} \nu_{j} & =h\left(\theta-\theta_{a}\right) \quad \text { on } \quad \Gamma:=\partial \Omega \times P, \\
\varphi(x, t) & =\varphi_{0}(x, t) \quad \text { on } \quad \Sigma_{D}:=\Gamma_{D} \times P, \\
\frac{\partial \varphi(x, t)}{\partial \nu} & =0 \quad \text { on } \quad \Sigma_{N}:=\Gamma_{N} \times P, \\
\mathbf{u}(x, t) & =0 \quad \text { on } \quad \Sigma_{D}, \\
\frac{\partial \mathbf{u}(x, t)}{\partial \nu} & =0 \quad \text { on } \quad \Sigma_{N}, \\
\theta(x, t+\omega) & =\theta(x, t), \quad \varphi(x, t+\omega)=\varphi(x, t), \\
\mathbf{u}(x, t+\omega) & =\mathbf{u}(x, t) \quad \text { in } \quad Q, \omega>0 .
\end{aligned}
$$

The coefficient of heat exchange $h$ is positive and the temperature near $\partial \Omega$ is denoted by $\theta_{a}$. Moreover, we assume that the body is held fixed on $\Sigma_{D}$ i.e. $\mathbf{u}=\mathbf{0}$ while on $\Sigma_{N}$, the body is free. The conditions on the boundary hold in the trace sense.

We observe that if

$$
\varphi \in L^{2}\left(P ; W^{1,2}(\Omega)\right) \cap L^{\infty}(Q),
$$

the equation $(1.1)_{2}$ allows to compute

$$
\sigma(\theta)|\nabla \varphi|^{2}=\operatorname{div}(\sigma(\theta) \varphi \nabla \varphi)
$$

in the sense of distributions.

Therefore, we will establish the existence of periodic solutions for the problem

$$
\left\{\begin{array}{c}
\theta_{t}-\sum_{i, j=1}^{n} \frac{\partial}{\partial x_{j}}\left(k_{i j} \frac{\partial \theta}{\partial x_{i}}\right)=\operatorname{div}(\sigma(\theta) \varphi \nabla \varphi)-\sum_{i, j=1}^{n} m_{i j} \frac{\partial^{2} u_{i}}{\partial t \partial x_{j}} \text { in } Q, \\
\operatorname{div}(\sigma(\theta) \nabla \varphi)=0 \text { in } Q, \\
-\sum_{i, j, k, l=1}^{n} \frac{\partial}{\partial x_{j}}\left(a_{i j k l} \frac{\partial u_{k}}{\partial x_{l}}\right)+\sum_{i, j=1}^{n} \frac{\partial}{\partial x_{j}}\left(m_{i j} \theta\right)=f_{i} \text { in } Q,
\end{array}\right\}
$$

with boundary and periodic conditions (1.2)-(1.5).

The unknown of system are the temperature $\theta(x, t)$ inside the conductor $\Omega$, the electrical potential $\varphi(x, t)$ and the displacement vector field $\mathbf{u}(x, t)=$ $\left(u_{1}(x, t), \ldots, u_{n}(x, t)\right)$. 
Thermistors are electrical devices of thermally sensitive resistors extensively utilized in industrial world. They have received much attention in the mathematical literature (see, e.g. $[1,2,5-11,18,19]$ ). In [20], is showed the existence of "capacity solutions" for the thermistor problem with the addition of dynamic thermoelastic equations containing terms of the form $\rho \frac{\partial^{2} u_{i}}{\partial t^{2}}$. When $\rho \frac{\partial^{2} u_{i}}{\partial t^{2}}$ may be omitted, the resulting problem is quasi-static. Quasi-static thermoelastic systems have been widely considered in literature. For more details we refer to the works $[12,16,17]$ and the references therein. However, in all these references the periodic case was not studied. The motivation for considering the quasi-static thermoelastic thermistor, arises from the often cracked and broken for these devices because of the thermal stresses.

In this paper, the periodic solutions for the new thermistor problem are seeked in suitable spaces of $\omega$-periodic functions without to look for fixed points to the periodic Poincaré map. Methodologically, we employ the functional frame of some results on the maximal monotone mappings.

To carry out this kind of program, the starting point relies on the possibility to reformulate our system as an abstract problem to which one applies some techniques deriving from the maximal monotone mappings theory and to get periodic solutions as a fixed point of an operator equation.

The plan of paper is the following: In Sections 2 and 3 we give some preliminaries and resolve the stationary charge equation of electrical current when we substitute $\sigma(\theta)$ by $\sigma(\zeta)$, for $\zeta \in L^{2}(Q)$. Next, we pass to treat the thermoelastic equations and the nonlinear heat equation in Sections 4 and 5 . Finally, Section 6 is devoted to prove the existence and uniqueness of weak periodic solutions $\theta_{n}$, $\varphi_{n}, \mathbf{u}_{n}$ for the approximating problems obtained replacing $\sigma(\zeta)$ by $\sigma\left(\zeta_{n}\right)$ and $\zeta_{n}$ $\in L^{2}(Q)$ in place of $\theta$ in (2.3) below. Furthemore, are deduced necessary uniform a priori estimates which will play a foundamental role joint to a fixed point argument, to infer the existence of weak periodic solutions for system (1.1')-(1.5) as limit of the approximating solutions $\theta_{n}, \varphi_{n}, \mathbf{u}_{n}$.

\section{Preliminaries}

Next, let us introduce the functional framework for the periodic solutions of problem. To this end, we consider the functional spaces

$$
\begin{gathered}
W_{D}^{1,2}(\Omega):=\left\{v \in W^{1,2}(\Omega): v=0 \text { on } \Gamma_{D}\right\}, \\
\left(W_{D}^{1,2}(\Omega)\right)^{n}:=\left\{v \in\left(W^{1,2}(\Omega)\right)^{n}: v=0 \text { on } \Gamma_{D}\right\}
\end{gathered}
$$

and a triplet of Hilbert spaces

$$
\begin{aligned}
V_{D} & :=L^{2}\left(P ; W_{D}^{1,2}(\Omega)\right), \\
V & :=L^{2}\left(P ; W^{1,2}(\Omega)\right), \\
W & :=L^{2}\left(P ;\left(W_{D}^{1,2}(\Omega)\right)^{n}\right),
\end{aligned}
$$


endowed with the respective norms

$$
\begin{aligned}
\|v\|_{V_{D}} & :=\left(\int_{Q}|v(x, t)|^{2} d x d t+\int_{Q}|\nabla v(x, t)|^{2} d x d t\right)^{1 / 2} \\
\|v\|_{V} & :=\left(\int_{Q}|\nabla v(x, t)|^{2} d x d t+\int_{\Gamma}|v(x, t)|^{2} d s d t\right)^{1 / 2}
\end{aligned}
$$

and

$$
\|\mathbf{u}\|_{W}:=\left(\sum_{i=1}^{n} \int_{Q}\left|u_{i}(x, t)\right|^{2} d x d t+\sum_{i=1}^{n} \int_{Q}\left|\nabla u_{i}(x, t)\right|^{2} d x d t\right)^{1 / 2} .
$$

The topological dual spaces of $V_{D}, V$ and $W$ shall be denoted by $V_{D}^{*}, V^{*}$ and $W^{*}$ respectively, with $\|\cdot\|_{*}$ norm. The pairing of duality between these spaces shall be written as $\langle.,$.$\rangle .$

Remark 2.1. We note that the norm in $V$ is equivalent to

$$
\left(\int_{Q}|v(x, t)|^{2} d x d t+\int_{Q}|\nabla v(x, t)|^{2} d x d t\right)^{1 / 2}
$$

To study our system we will do the following structural assumptions on the data

$\left.\mathrm{H}_{\sigma}\right) \sigma \in C(R)$ such that $0<\sigma_{*} \leq \sigma(s) \leq \sigma^{*}, \forall s \in R$;

$\left.\mathrm{H}_{K}\right) k_{i j} \in L^{\infty}(Q)$ and $\exists \mu>0: \sum_{i, j=1}^{n} k_{i j} \xi_{i} \xi_{j} \geq \mu|\xi|^{2}, \forall \xi \in R^{n}$;

$\left.\mathrm{H}_{M}\right) m_{i j}, \frac{\partial m_{i j}}{\partial t} \in W^{1, \infty}(Q), m_{i j}=m_{j i}>0$;

$\left.\mathrm{H}_{A}\right)\left\{\begin{array}{c}a_{i j k l} \in L^{\infty}(Q), a_{i j k l}=a_{j i k l}=a_{k l i j}, \forall i, j, k, l \text { and } \\ \exists \lambda>0: \sum_{i, j, k, l=1}^{n} a_{i j k l} \eta_{i j} \eta_{k l} \geq \lambda \sum_{i, j=1}^{n}\left|\eta_{i j}\right|^{2}, \forall \eta=\left(\eta_{i j}\right), \eta_{i j}=\eta_{j i} ;\end{array}\right\}$

$\left.\mathrm{H}_{\mathbf{f}}\right) \mathbf{f} \in\left(L^{2}(Q)\right)^{n}$;

$\left.\mathrm{H}_{0}\right) \varphi_{0}$ is a $t$-periodic bounded function with an extention in $Q, \widetilde{\varphi}_{0} \in$ $L^{\infty}\left(P ; W^{1, \infty}(\Omega)\right)$ and $\frac{\partial \widetilde{\varphi}_{0}}{\partial \nu}=0$ on $\Gamma_{D}$.

To complete the presentation of the quasi-static problem, we give the notion of weak solution.

Definition 2.2. A triplet $\{\theta, \varphi, \mathbf{u}\}$ is said to be a weak periodic solution of problem $\left(1.1^{\prime}\right)-(1.5)$ if satisfies

$$
\begin{aligned}
\theta \in L^{2}\left(P ; W^{1,2}(\Omega)\right), \quad \theta_{t} & \in L^{2}\left(P ;\left(W^{1,2}(\Omega)\right)^{*}\right) \\
\varphi-\varphi_{0} & \in L^{2}\left(P ; W_{D}^{1,2}(\Omega)\right) \\
\mathbf{u} \in L^{2}\left(P ;\left(W_{D}^{1,2}(\Omega)\right)^{n}\right), \quad \mathbf{u}_{t} & \in L^{2}\left(P ;\left(W^{-1,2}(\Omega)\right)^{n}\right)
\end{aligned}
$$


and

$$
\begin{aligned}
& \int_{Q} \theta_{t} w d x d t+\sum_{i, j=1}^{n} \int_{Q} k_{i j} \frac{\partial \theta}{\partial x_{i}} \frac{\partial w}{\partial x_{j}} d x d t+\int_{\Gamma} h\left(\theta-\theta_{a}\right) w d s d t \\
& =-\int_{Q} \sigma(\theta) \varphi \nabla \varphi \nabla w d x d t+\sum_{i, j=1}^{n} \int_{Q} \frac{\partial m_{i j}}{\partial t} \frac{\partial u_{i}}{\partial x_{j}} w d x d t, \quad \forall w \in V \\
& \int_{Q} \sigma(\theta) \nabla \varphi \nabla \xi d x d t=0, \quad \forall \xi \in V_{D} \\
& \sum_{i, j, k, l=1}^{n} \int_{Q} a_{i j k l} \frac{\partial u_{k}}{\partial x_{l}} \frac{\partial \rho_{i}}{\partial x_{j}} d x d t-\sum_{i, j=1}^{n} \int_{Q} m_{i j} \theta \frac{\partial \rho_{i}}{\partial x_{j}} d x d t \\
& =\int_{Q} f_{i} \rho_{i} d x d t, \quad \forall \rho \in W .
\end{aligned}
$$

\section{The stationary conservation of electrical current problem}

We begin showing the existence of weak periodic solutions for the stationary conservation of electrical current problem. Fixed $\zeta \in L^{2}(Q)$ and defined $z=\varphi-\varphi_{0}$, then this function should satisfy

$$
\begin{aligned}
\operatorname{div}\left(\sigma(\zeta)\left(\nabla z+\nabla \varphi_{0}\right)\right) & =0 \quad \text { in } \quad Q, \\
z(x, t) & =0 \quad \text { on } \quad \Sigma_{D}, \\
\frac{\partial z(x, t)}{\partial \nu} & =0 \quad \text { on } \quad \Sigma_{N}, \\
z(x, t+\omega) & =z(x, t) \quad \text { in } \quad Q, \quad \omega>0 .
\end{aligned}
$$

Definition 3.1. A weak periodic solution to (3.1)-(3.4) is a function

$$
z \in L^{2}\left(P ; W_{D}^{1,2}(\Omega)\right)
$$

such that

$$
\int_{Q} \sigma(\zeta)\left(\nabla z+\nabla \varphi_{0}\right) \nabla \xi d x d t=0, \quad \forall \xi \in V_{D}
$$

In our setting, the existence of solutions for (3.5) can be deduced from the next result.

Theorem 3.1 $([3,4,14])$. . If $A$ is a monotone, hemicontinuous mapping from $V_{D}$ to $V_{D}^{*}$ such that $A$ is coercitive, then Range $(A)=V_{D}^{*}$.

To proceed, we define the mapping A by setting

$$
\langle A z, \xi\rangle:=\int_{Q} \sigma(\zeta)\left(\nabla z+\nabla \varphi_{0}\right) \nabla \xi d x d t, \forall z, \quad \xi \in V_{D} .
$$

The properties of the mapping $A$ are given in the following result.

Proposition 3.2. Under assumptions $H_{\sigma}$ ) and $\left.H_{0}\right)$, the mapping $A$ is: 
i) hemicontinuous;

ii) monotone;

iii) coercive.

Proof. i) By the Hölder inequality, we infer that

$$
\begin{aligned}
|\langle A z, \xi\rangle| & \leq \sigma^{*}\left(\int_{Q}\left|\nabla z+\nabla \varphi_{0}\right|^{2} d x d t\right)^{1 / 2}\left(\int_{Q}|\nabla \xi|^{2} d x d t\right)^{1 / 2} \\
& \leq \sigma^{*} \sqrt{2}\left(\|z\|_{V_{D}}+\left\|\nabla \varphi_{0}\right\|_{L^{2}(Q)}\right)\|\xi\|_{V_{D}}
\end{aligned}
$$

by which

$$
\|A z\|_{*} \leq \sigma^{*} \sqrt{2}\left(\|z\|_{V_{D}}+\left\|\nabla \varphi_{0}\right\|_{L^{2}(Q)}\right) .
$$

The hemicontinuity is deduced from a result of [13, Theorems 2.1 and 2.3].

ii) $\left\langle A z_{1}-A z_{2}, z_{1}-z_{2}\right\rangle=\int_{Q} \sigma(\zeta)\left|\nabla\left(z_{1}-z_{2}\right)\right|^{2} d x d t \geq 0$.

iii) By the Poincaré inequality, for some constant $c$, one has

$$
\langle A z, z\rangle=\int_{Q} \sigma(\zeta)|\nabla z|^{2} d x d t \geq \sigma_{*} c\|z\|_{V_{D}}^{2}-\sigma^{*}\left\|\nabla \varphi_{0}\right\|_{L^{2}(Q)}\|z\|_{V_{D}}
$$

so that

$$
\frac{\langle A z, z\rangle}{\|z\|_{V_{D}}} \geq \sigma_{*} c\|z\|_{V_{D}}-\sigma^{*}\left\|\nabla \varphi_{0}\right\|_{L^{2}(Q)} \rightarrow+\infty \quad \text { as } \quad\|z\|_{V_{D}} \rightarrow+\infty .
$$

Thus, the equivalence between (3.5) and the abstract problem

$$
A z=0 \text {. }
$$

is readly understood.

Hence, we can state the main result for the stationary consevation of electrical current problem.

Theorem 3.3. Let $\left.H_{\sigma}\right)$ and $\left.H_{0}\right)$ be satisfied, there exists a unique periodic solution to $(3.6)$.

Proof. The existence of solutions is a consequence of Theorem 3.1. The uniqueness descends from the strict monotonicity of the mapping $A$.

Therefore, in correspondence of a fixed $\zeta \in L^{2}(Q)$, has been proved the existence and uniqueness of the weak periodic solution $\varphi$ for the problem

$$
\begin{aligned}
\operatorname{div}(\sigma(\zeta) \nabla \varphi) & =0 \quad \text { in } \quad Q, \\
\varphi(x, t) & =\varphi_{0}(x, t) \quad \text { on } \quad \Sigma_{D}:=\Gamma_{D} \times P, \\
\frac{\partial \varphi(x, t)}{\partial \nu}=0 & \text { on } \quad \Sigma_{N}:=\Gamma_{N} \times P, \\
\varphi(x, t+\omega) & =\varphi(x, t), \quad \text { in } \quad Q, \omega>0,
\end{aligned}
$$

Since $\widetilde{\varphi}_{0} \in L^{\infty}\left(P ; W^{1, \infty}(\Omega)\right)$, by the weak maximum principle one obtains

$$
\|\varphi\|_{L^{\infty}(Q)} \leq e s s \sup _{Q}\left|\widetilde{\varphi}_{0}(x, t)\right|
$$


that is

$$
\varphi \in L^{2}\left(P ; W^{1,2}(\Omega)\right) \cap L^{\infty}(Q) .
$$

To establish the classical energy estimate, we multiply (3.7) by $\varphi-\varphi_{0}$ and integrate over $Q$. This yields

$$
\begin{aligned}
\sigma_{*} \int_{Q}|\nabla \varphi(x, t)|^{2} d x d t & \leq \int_{Q} \sigma(\zeta)|\nabla \varphi(x, t)|^{2} d x d t \\
& \leq \int_{Q} \sigma(\zeta) \nabla \varphi_{0} \nabla \varphi d x d t \\
& \leq \sigma^{*}\left(\int_{Q}|\nabla \varphi(x, t)|^{2} d x d t\right)^{1 / 2} \\
& \times\left(\int_{Q}\left|\nabla \varphi_{0}(x, t)\right|^{2} d x d t\right)^{1 / 2}
\end{aligned}
$$

hence

$$
\int_{Q}|\nabla \varphi(x, t)|^{2} d x d t \leq\left(\frac{\sigma^{*}}{\sigma_{*}}\right)^{2}\left\|\nabla \varphi_{0}\right\|_{L^{2}(Q)}^{2} .
$$

Combining (3.11) and (3.12) one deduces that

$$
\int_{Q}|\varphi(x, t)|^{2} d x d t+\int_{Q}|\nabla \varphi(x, t)|^{2} d x d t \leq N .
$$

Here and in the sequel, $N>0$ denotes various different constants.

\section{Thermoelastic systems}

Now, turn our interest to investigate the problem

$$
\begin{aligned}
\sum_{i, j, k, l=1}^{n} \int_{Q} a_{i j k l} \frac{\partial u_{k}}{\partial x_{l}} \frac{\partial \rho_{i}}{\partial x_{j}} d x d t-\sum_{i, j=1}^{n} \int_{Q} m_{i j} \zeta \frac{\partial \rho_{i}}{\partial x_{j}} d x d t \\
\quad=\int_{Q} f_{i} \rho_{i} d x d t, \quad \forall \rho \in W .
\end{aligned}
$$

According to Theorem 3.1, let define the mapping $B: W \rightarrow W^{*}$ for any $\mathbf{u} \in W$ as

$$
\langle B \mathbf{u}, \rho\rangle:=\sum_{i, j, k, l=1}^{n} \int_{Q} a_{i j k l} \frac{\partial u_{k}}{\partial x_{l}} \frac{\partial \rho_{i}}{\partial x_{j}} d x d t, \quad \forall \rho \in W .
$$

It is easy to check that $B$ verifies the same statements i)-iii) in Proposition 3.2. Indeed,

Proposition 4.1. If $\left.\left.H_{A}\right)-H_{\mathbf{f}}\right)$ are fulfilled then, the mapping $B$ satisfies the properties i)-iii) of Proposition 3.2. 
Proof. i) One has

$$
\begin{aligned}
|\langle B \mathbf{u}, \rho\rangle| \leq & \left(\text { ess } \sup _{Q}\left|a_{i j k l}(x, t)\right|\right)\left(\int_{Q}\left|\frac{\partial u_{k}}{\partial x_{l}}(x, t)\right|^{2} d x d t\right)^{1 / 2} \\
& \times\left(\int_{Q}\left|\frac{\partial \rho_{i}}{\partial x_{j}}(x, t)\right|^{2} d x d t\right)^{1 / 2} \\
\leq & \left(e s s \sup _{Q}\left|a_{i j k l}(x, t)\right|\right)\|\mathbf{u}\|_{W}\|\rho\|_{W},
\end{aligned}
$$

hence

$$
\|B \mathbf{u}\|_{*} \leq\left(\operatorname{ess} \sup _{Q}\left|a_{i j k l}(x, t)\right|\right)\|\mathbf{u}\|_{W} .
$$

From [13, Theorems 2.1 and 2.3] we obtain the hemicontinuity.

ii) From $\mathrm{H}_{A}$ ), we have

$$
\left\langle B \mathbf{u}_{1}-B \mathbf{u}_{2}, \mathbf{u}_{1}-\mathbf{u}_{2}\right\rangle=\sum_{i, j, k, l=1}^{n} \int_{Q} a_{i j k l} \frac{\partial\left(u_{k}^{1}-u_{k}^{2}\right)}{\partial x_{l}} \frac{\partial\left(u_{i}^{1}-u_{i}^{2}\right)}{\partial x_{j}} d x d t \geq 0 .
$$

iii) On account of the inequality of Poincaré, we infer that

$$
\begin{aligned}
\langle B \mathbf{u}, \mathbf{u}\rangle & =\sum_{i, j, k, l=1}^{n} \int_{Q} a_{i j k l} \frac{\partial u_{k}}{\partial x_{l}} \frac{\partial u_{i}}{\partial x_{j}} d x d t \\
& \geq \lambda \sum_{i, j=1}^{\infty} \int_{Q}\left|\frac{\partial u_{i}}{\partial x_{j}}\right|^{2} d x d t \geq \lambda c\|\mathbf{u}\|_{W}^{2},
\end{aligned}
$$

therefore

$$
\frac{\langle B \mathbf{u}, \mathbf{u}\rangle}{\|\mathbf{u}\|_{W}} \geq \lambda c\|\mathbf{u}\|_{W} \rightarrow+\infty, \quad \text { as } \quad\|\mathbf{u}\|_{W} \rightarrow+\infty
$$

A solution of (4.1) will be obtained by studying the equivalent abstract problem.

$$
B \mathbf{u}=H
$$

where $H \in W^{*}$ is so defined

$$
\langle H, \rho\rangle:=\sum_{i, j=1}^{n} \int_{Q} m_{i j} \zeta \frac{\partial \rho_{i}}{\partial x_{j}} d x d t+\int_{Q} f_{i} \rho_{i} d x d t, \quad \forall \rho \in W .
$$

Because of Theorem 3.1 and the strict monotonicity of the mapping $B$, we are able to state the main result for the quasi-static thermoelastic problem affirming that

Proposition 4.2. Given a $\zeta \in L^{2}(Q)$, assuming $\left.\left.H_{A}\right)-H_{\mathbf{f}}\right)$ the problem (4.2) admits a unique periodic solution. 
Taking as a test function $\mathbf{u}$ in (4.1), we get

$$
\begin{aligned}
\lambda \int_{Q}|\nabla \mathbf{u}(x, t)|^{2} d x d t & \leq \sum_{i, j, k, l=1}^{n} \int_{Q} a_{i j k l} \frac{\partial u_{k}}{\partial x_{l}} \frac{\partial u_{i}}{\partial x_{j}} d x d t \\
& =\sum_{i, j=1}^{n} \int_{Q} m_{i j} \zeta \frac{\partial u_{i}}{\partial x_{j}} d x d t+\int_{Q} f_{i} u_{i} d x d t
\end{aligned}
$$

and by Poincaré's inequality one checks that

$$
\lambda \int_{Q}|\nabla \mathbf{u}(x, t)|^{2} d x d t \leq\left(\left(\text { ess } \sup _{Q} m_{i j}\right)\|\zeta\|_{L^{2}(Q)}+c\|\mathbf{f}\|_{L^{2}(Q)} \|\right)\|\nabla \mathbf{u}\|_{L^{2}(Q)} .
$$

Hence,

$$
\int_{Q}|\nabla \mathbf{u}(x, t)|^{2} d x d t \leq\left(\frac{\left(\left(e s s \sup _{Q} m_{i j}\right)\|\zeta\|_{L^{2}(Q)}+c\|\mathbf{f}\|_{L^{2}(Q)} \|\right)}{\lambda}\right)^{2}
$$

and the classical energy estimate is established.

$$
\int_{Q}|\mathbf{u}(x, t)|^{2} d x d t+\int_{Q}|\nabla \mathbf{u}(x, t)|^{2} d x d t \leq N .
$$

\section{Nonlinear energy heat flow}

Remain to take into consideration the heat conduction problem

$$
\begin{array}{rl}
\int_{Q} \theta_{t} w & d x d t+\sum_{i, j=1}^{n} \int_{Q} k_{i j} \frac{\partial \theta}{\partial x_{i}} \frac{\partial w}{\partial x_{j}} d x d t+\int_{\Gamma} h\left(\theta-\theta_{a}\right) w d s d t \\
=-\int_{Q} \sigma(\zeta) \varphi \nabla \varphi \nabla w d x d t+\sum_{i, j=1}^{n} \int_{Q} \frac{\partial m_{i j}}{\partial t} \frac{\partial u_{i}}{\partial x_{j}} w d x d t, \quad \forall w \in V .
\end{array}
$$

The existence of weak periodic solutions to (5.1) is based on the following result

Theorem $5.1([3,4,14])$. . Let $L$ be a linear closed densely defined operator from the reflexive Banach space $V$ to $V^{*}, L$ maximal monotone and let $C$ be a bounded, hemicontinuous monotone mapping from $V$ into $V^{*}$. Moreover, if $L+C$ is coercitive then the Range $(L+C)=V^{*}$.

In order to use Theorem 5.1, we must define the operators $L$ and $C$. The set

$$
D:=\left\{\theta \in L^{2}\left(P ; W^{1,2}(\Omega)\right) ; \theta_{t} \in L^{2}\left(P ; W^{1,2}(\Omega)^{*}\right)\right\}
$$

is dense in $V$ because of the density of $C(\bar{Q}) \subset D$ in $V$.

Let

$$
L: D \rightarrow V^{*}
$$


be the linear defined by

$$
\langle L \theta, w\rangle:=\int_{Q} \theta_{t} w d x d t, \quad \forall w \in V .
$$

This operator $L$ is closed, skew-adjoint (i.e. $L=-L^{*}$ ) and maximal monotone (see [14, Lemma 1.1, p. 313]).

As operator $C$ we take

$$
\langle C \theta, w\rangle:=\sum_{i, j=1}^{n} \int_{Q} k_{i j} \frac{\partial \theta}{\partial x_{i}} \frac{\partial w}{\partial x_{j}} d x d t+\int_{\Gamma} h \theta w d s d t, \quad \forall w \in V .
$$

The properties of $C$ are resumed in the next result

Proposition 5.2. If assumptions $\left.H_{\sigma}\right)-H_{M}$ ) are satisfied, the operator $C$ is

i) hemicontinuous;

ii) monotone;

iii) coercive.

Proof. i) Applying Hölder's inequality one proves that

$$
\begin{aligned}
& |\langle C \theta, w\rangle| \leq\left(\text { ess } \sup _{Q}\left|k_{i j}(x, t)\right|\right) \\
& \times\left(\int_{Q}\left|\frac{\partial \theta}{\partial x_{i}}\right|^{2} d x d t\right)^{1 / 2}\left(\int_{Q}\left|\frac{\partial w}{\partial x_{j}}\right|^{2} d x d t\right)^{1 / 2} \\
& +h\left(\int_{\Gamma}|\theta|^{2} d s d t\right)^{1 / 2}\left(\int_{\Gamma}|w|^{2} d s d t\right)^{1 / 2} \\
& \leq\left(e s s \sup _{Q}\left|k_{i j}(x, t)\right|+h\right)\|\theta\|_{V}\|w\|_{V}
\end{aligned}
$$

thus,

$$
\|C \theta\|_{*} \leq\left(\text { ess } \sup _{Q}\left|k_{i j}(x, t)\right|+h\right)\|\theta\|_{V}
$$

and the hemicontinuity is a consequence of a result of [13, Theorems 2.1 and 2.3].

ii)

$$
\begin{aligned}
\left\langle C \theta_{1}-C \theta_{2}, \theta_{1}-\theta_{2}\right\rangle= & \sum_{i, j=1}^{n} \int_{Q} k_{i j} \frac{\partial\left(\theta_{1}-\theta_{2}\right)}{\partial x_{i}} \frac{\partial\left(\theta_{1}-\theta_{2}\right)}{\partial x_{j}} d x d t \\
& +\int_{\Gamma} h\left(\theta_{1}-\theta_{2}\right)^{2} d s d t \\
\geq & \mu \int_{Q}\left|\nabla\left(\theta_{1}-\theta_{2}\right)\right|^{2} d x d t \\
& +\int_{\Gamma} h\left(\theta_{1}-\theta_{2}\right)^{2} d s d t \geq 0 .
\end{aligned}
$$


iii)

$$
\begin{aligned}
\langle C \theta, \theta\rangle & =\sum_{i, j=1}^{n} \int_{Q} k_{i j} \frac{\partial \theta}{\partial x_{i}} \frac{\partial \theta}{\partial x_{j}} d x d t+h \int_{\Gamma}|\theta|^{2} d s d t \\
& \geq \mu \int_{Q}|\nabla \theta|^{2} d x d t+h \int_{\Gamma}|\theta|^{2} d s d t \min \{\mu, h\}\|\theta\|_{V}^{2}
\end{aligned}
$$

Consequently,

$$
\frac{\langle C \theta, \theta\rangle}{\|\theta\|_{V}} \geq \min \{\mu, h\}\|\theta\|_{V} \rightarrow+\infty, \quad \text { as } \quad\|\theta\|_{V} \rightarrow+\infty
$$

We point out that to apply Theorem 5.1, we must reformulate problem (5.1) in abstract form. Let define $G \in V^{*}$ by setting

$$
\begin{aligned}
\langle G, w\rangle= & -\int_{Q} \sigma(\zeta) \varphi \nabla \varphi \nabla w d x d t+\sum_{i, j=1}^{n} \int_{Q} \frac{\partial m_{i j}}{\partial t} \frac{\partial u_{i}}{\partial x_{j}} w d x d t \\
& +\int_{\Gamma} h \theta_{a} w d s d t
\end{aligned}
$$

then, problem (5.1) can be handled as the abstract problem

$$
L \theta+C \theta=G
$$

that admits a unique periodic solution, thanks to Theorem 5.1 and the strict monotonicity of $C$.

Now, we derive a priori estimate for $\theta$. The chosen of $\theta$ as a test function in (5.1) gives us

$$
\begin{aligned}
\mu \int_{Q}|\nabla \theta|^{2} d x d t+\int_{\Gamma} h\left(\theta-\theta_{a}\right) \theta d s d t \leq & \sum_{i, j=1}^{n} \int_{Q} k_{i j} \frac{\partial \theta}{\partial x_{i}} \frac{\partial \theta}{\partial x_{j}} d x d t \\
& +\int_{\Gamma} h\left(\theta-\theta_{a}\right) \theta d s d t \\
= & -\int_{Q} \sigma(\zeta) \varphi \nabla \varphi \nabla \theta d x d t \\
& +\sum_{i, j=1}^{n} \int_{Q} \frac{\partial m_{i j}}{\partial t} \frac{\partial u_{i}}{\partial x_{j}} \theta d x d t
\end{aligned}
$$


which leads to

$$
\begin{aligned}
\mu \int_{Q}|\nabla \theta|^{2} d x d t+h \int_{\Gamma}|\theta|^{2} d s d t \\
\leq \sigma^{*}\|\varphi\|_{L^{\infty}(Q)}\left(\int_{Q}|\nabla \varphi(x, t)|^{2} d x d t\right)^{1 / 2}\left(\int_{Q}|\nabla \theta|^{2} d x d t\right)^{1 / 2} \\
\quad+h\left(\int_{\Gamma}\left|\theta_{a}\right|^{2} d s d t\right)^{1 / 2}\left(\int_{\Gamma}|\theta|^{2} d s d t\right)^{1 / 2} \\
\quad+e s s \sup _{Q}\left|\frac{\partial m_{i j}}{\partial t}\right|\left(\int_{Q}|\nabla \mathbf{u}(x, t)|^{2} d x d t\right)^{1 / 2}\left(\int_{Q}|\theta|^{2} d x d t\right)^{1 / 2}
\end{aligned}
$$

The equivalence of the norm in $V$, yields

$$
\int_{Q}|\nabla \theta|^{2} d x d t+\int_{\Gamma}|\theta|^{2} d s d t \leq N
$$

\section{A fixed point argument}

The periodicity of weak solutions to $\left(1.1^{\prime}\right)-(1.5)$ is proved by means of a topological argument which is the appropriate tool to research fixed points for a suitable operator equation. To this end, let

$$
\Phi: L^{2}(Q) \rightarrow L^{2}(Q)
$$

be the nonlinear mapping defined by

$$
\Phi(\zeta)=\theta
$$

where $\theta$ is the unique weak periodic solution to (5.1). The mapping $\Phi$ is welldefined. In order to prove its continuity, we prove some crucial estimates and convergences, needed to utilize the Schauder fixed point theorem. Let $\zeta_{n} \in L^{2}(Q)$ be a sequence such that $\zeta_{n} \rightarrow \zeta$ and $\sigma\left(\zeta_{n}\right) \rightarrow \sigma(\zeta)$ strongly in $L^{2}(Q)$, we denote with $\theta_{n}, \varphi_{n}, \mathbf{u}_{n}$ respectively, the weak periodic solutions of

$$
\begin{aligned}
& \int_{Q} \theta_{n t} w d x d t+\sum_{i, j=1}^{n} \int_{Q} k_{i j} \frac{\partial \theta_{n}}{\partial x_{i}} \frac{\partial w}{\partial x_{j}} d x d t+\int_{\Gamma} h\left(\theta_{n}-\theta_{a}\right) w d s d t \\
& =-\int_{Q} \sigma\left(\zeta_{n}\right) \varphi_{n} \nabla \varphi_{n} \nabla w d x d t+\sum_{i, j=1}^{n} \int_{Q} \frac{\partial m_{i j}}{\partial t} \frac{\partial u_{n i}}{\partial x_{j}} w d x d t, \quad \forall w \in V . \\
& \int_{Q} \sigma\left(\zeta_{n}\right) \nabla \varphi_{n} \nabla \xi d x d t=0, \quad \forall \xi \in V_{0}
\end{aligned}
$$


and

$$
\begin{aligned}
\sum_{i, j, k, l=1}^{n} \int_{Q} a_{i j k l} \frac{\partial u_{n k}}{\partial x_{l}} \frac{\partial \rho_{i}}{\partial x_{j}} d x d t-\sum_{i, j=1}^{n} \int_{Q} m_{i j} \zeta_{n} \frac{\partial \rho_{i}}{\partial x_{j}} d x d t & \\
& =\int_{Q} f_{i} \rho_{i} d x d t, \quad \forall \rho \in W .
\end{aligned}
$$

Arguing as previously, we get the estimates

$$
\begin{gathered}
\left\|\varphi_{n}\right\|_{L^{\infty}(Q)} \leq \operatorname{sessp}_{Q}\left|\widetilde{\varphi}_{0}(x, t)\right| \\
\int_{Q}\left|\varphi_{n}(x, t)\right|^{2} d x d t+\int_{Q}\left|\nabla \varphi_{n}(x, t)\right|^{2} d x d t \leq N \\
\int_{Q}\left|\nabla \theta_{n}\right|^{2} d x d t+\int_{\Gamma}\left|\theta_{n}\right|^{2} d s d t \leq N \\
\int_{Q}\left|\mathbf{u}_{n}(x, t)\right|^{2} d x d t+\int_{Q}\left|\nabla \mathbf{u}_{n}(x, t)\right|^{2} d x d t \leq N .
\end{gathered}
$$

In this section, $N>0$ denotes various different constants independent of $n$.

Moreover,

Lemma 6.1. The sequence $\nabla \varphi_{n}$ converges strongly to $\nabla \varphi$ in $L^{2}\left(P ;\left(L^{2}(\Omega)\right)^{n}\right)$.

Proof. Choosing $\varphi_{n}-\varphi$ as test function in (6.2), we have

So

$$
\int_{Q} \sigma\left(\zeta_{n}\right)\left|\nabla\left(\varphi_{n}-\varphi\right)\right|^{2} d x d t=\int_{Q} \sigma\left(\zeta_{n}\right) \nabla \varphi \nabla\left(\varphi_{n}-\varphi\right) d x d t
$$

$$
\sigma_{*} \int_{Q}\left|\nabla\left(\varphi_{n}-\varphi\right)\right|^{2} d x d t \leq \int_{Q} \sigma\left(\zeta_{n}\right) \nabla \varphi \nabla\left(\varphi_{n}-\varphi\right) d x d t .
$$

The weak convergence of $\sigma\left(\zeta_{n}\right) \nabla\left(\varphi_{n}-\varphi\right)$ to zero as $n \rightarrow+\infty$, leads to the conclusion.

Lemma 6.1, allows to obtain the convergence

$$
\varphi_{n} \rightarrow \varphi \text { in } L^{2}\left(P ;\left(L^{2}(\Omega)\right)^{n}\right) \text { and a.e. in } Q .
$$

By virtue of (6.1) and (6.7), $\theta_{n t}$ is bounded with respect to the norm of $V^{*}$. This ensures that $\theta_{n}$ belongs to a bounded set of $D$

$$
\left\|\theta_{n}\right\|_{D} \leq C .
$$

Thus, we can select a subsequence, still denoted by $\theta_{n}$, such that

$$
\theta_{n} \rightarrow \theta \text { in } D \text {. }
$$

By a result of $\left[14\right.$, Theorem 5.1], the sequence $\theta_{n}$ is precompact in $L^{2}(Q)$ that is

$$
\theta_{n} \rightarrow \theta \text { in } L^{2}(Q) \text { and a.e. in } Q
$$

Lemma 6.2. The mapping $\Phi$ is continuous. 
Proof. The previous results, imply the following convergences for a suitable chosen subsequence

$$
\begin{aligned}
\nabla \theta_{n} & \rightarrow \nabla \theta \quad \text { in } \quad L^{2}\left(P ;\left(L^{2}(\Omega)\right)^{n}\right) \\
\nabla u_{n i} & \rightarrow \nabla u_{i} \quad \text { in } \quad L^{2}\left(P ;\left(L^{2}(\Omega)\right)^{n}\right) \\
\nabla \varphi_{n} & \rightarrow \nabla \varphi \quad \text { in } \quad L^{2}\left(P ;\left(L^{2}(\Omega)\right)^{n}\right) \\
\zeta_{n} & \rightarrow \zeta \quad \text { in } \quad L^{2}(Q) \\
\sigma\left(\zeta_{n}\right) & \rightarrow \sigma(\zeta) \quad \text { in } \quad L^{2}(Q)
\end{aligned}
$$

and according to the trace theorem (see [15, Theorem 3.4.5]), one obtains

$$
\theta_{n} \rightarrow \theta \quad \text { in } L^{2}\left(P ; L^{2}(\partial \Omega)\right) .
$$

Therefore, the assertion of lemma descends from the above convergences since $\Phi\left(\zeta_{n}\right)=\theta_{n}$ converges strongly to $\Phi(\zeta)=\theta$ in $L^{2}(Q)$.

Lemma 6.3. There exists a constant $R>0$ such that

$$
\|\Phi(\zeta)\|_{L^{2}(Q)} \leq R, \quad \forall \zeta \in L^{2}(Q) .
$$

Proof. Letting the limit in (6.6), we have the conclusion.

Since $\Phi\left(L^{2}(Q)\right) \subset D$ and the embedding of $D \hookrightarrow L^{2}(Q)$ is compact, $\Phi$ is a compact mapping from $L^{2}(Q)$ into itself.

Now, only remains to state the main result of paper

Theorem 6.4. Let $\left.\left.H_{\sigma}\right)-H_{0}\right)$ be satisfied, there is at least a weak periodic solution to system $\left(1.1^{\prime}\right)-(1.5)$.

Proof. As a consequence of Lemmas 6.2 and 6.3 the mapping $\Phi$ is both continuous and compact. Hence, by the Schauder fixed point theorem $\Phi$ has as a fixed point which is a weak periodic solution for $\left(1.1^{\prime}\right)-(1.5)$. This conclude the proof.

\section{References}

[1] W. Alegretto, Y. Lin and S. Ma, On the time periodic thermistor problem, European J. Appl. Math. 15 (2004), no. 1, 55-77.

[2] S. N. Antonsev and M. Chipot, The thermistor problem: existence, smoothness, uniqueness, blowup, SIAM J. Math. Anal. 25 (1994), no. 4, 1128-1156.

[3] V. Barbu, Nonlinear semigroups and differential equations in Banach spaces, Noordhoff International Publishing Leyden The Netherlands 1976.

[4] F. E. Browder, Nonlinear maximal monotone operators in Banach space, Math. Ann. 175 (1968), 89-113.

[5] M. Chipot and G. Cimatti, A uniqueness result for the thermistor problem, European J. Appl. Math. 2 (1991), no. 2, 97-103.

[6] G. Cimatti, A bound for the temperature in the thermistor problem, IMA J. Appl. Math. 40 (1988), no. 1, 15-22. 
[7] G. Cimatti, Remark on existence and uniqueness for the thermistor problem under mixed boundary conditions, Quart. Appl. Math. 47 (1989), no. 1, 117-121.

[8] G. Cimatti, On the stability of the solution of the thermistor problem, Appl. Anal. 73 (1999), no. 3-4, 407-423.

[9] G. Cimatti, A remark on the thermistor problem with rapidly growing conductivity, Appl. Anal. 80 (2001), no. 1-2, 133-140.

[10] G. Cimatti, Stability and multiplicity of solutions for the thermistor problem, Ann. Mat. Pura Appl. (4) 181 (2002), no. 2, 181-212.

[11] G. Cimatti, Asymptotics for the time-dependent thermistor problem, Quart. Appl. Math. 62 (2004), no. 3, 471-476.

[12] R. P. Gilbert, P. Shi and M. Shillor, A quasi-static contact problem in linear thermoelasicity, Rendiconti di Matematica 10 (1990), 785-808.

[13] M. A. Krasnoselskii, Topological methods in the theory of nonlinear integral equations, Pergamon Press, New York 1964.

[14] J. L. Lions, Quelques méthode de résolution de problemes aux limites non-linéaires, Dunod Paris 1969.

[15] C. B. Morrey Jr., Multiple integrals in the calculus of variations, Springer Verlag, New York 1966.

[16] J. E. Muñoz Rivera and R. Racke, Multidimensional contact problems in thermoelasticity, SIAM J. Appl. Math. 58 (1998), no. 4, 1307-1337.

[17] P. Shi and M. Shillor, Existence of a solution to the $\mathrm{n}$ dimensional problem of thermoelastic contact, Commun. in Partial Differential equations, 17 (1992) no. 9-10, $1597-1618$.

[18] X. Xu, The thermistor problem with conductivity vanishing for large temperature. Proc. Roy. Soc. Edinburgh Sect. A 124 (1994), no. 1, 1-21.

[19] X. Xu, On the existence of bounded temperature in the thermistor problem with degeneracy, Nonlinear Anal. 42 (2000), no. 2, Ser. A: Theory Methods, 199-213.

[20] X. Wu and X. Xu, Existence for the thermoelastic thermistor problem, J. Math. Anal. Appl. 319 (2006), 214-138.

Maurizio Badii

Dipartimento di Matematica "G. Castelnuovo"

Università di Roma "La Sapienza"

P.le A. Moro 2

00185 Roma

Italy

e-mail: badii@mat.uniroma1.it

Received: 17 January 2007.

Accepted: 7 February 2008. 